Kragujevac Journal of Mathematics

Volume 42(1) (2018), Pages 97-106.

\title{
EXTREMAL VALUES OF MERRIFIELD-SIMMONS INDEX FOR TREES WITH TWO BRANCHING VERTICES
}

\author{
ROBERTO CRUZ ${ }^{1}$, CARLOS ALBERTO MARÍN ${ }^{2}$, AND JUAN RADA ${ }^{3}$
}

\begin{abstract}
In this paper we find trees with minimal and maximal MerrifieldSimmons index over the set $\Omega(n, 2)$ of all trees with $n$ vertices and 2 branching vertices, and also over the subset $\Omega^{t}(n, 2)$ of all trees in $\Omega(n, 2)$ such that the branching vertices are connected by the path $P_{t}$.
\end{abstract}

\section{INTRODUCTION}

A topological index is a numerical value associated to a molecular graph of a chemical compound, used for correlation of chemical structure with physical properties, chemical reactivity or biological activity $[2,9,10]$. Among the numerous topological indices considered in chemical graph theory, an important example is the MerrifieldSimmons index, conceived by the chemists Merrifield and Simmons for describing molecular structure by means of finite-set topology [7]. Given a graph $G$, denote by $n(G, k)$ the number of ways in which $k$ mutually independent vertices can be selected in $G$. By definition $n(G, 0)=1$ for all graphs, and $n(G, 1)$ is the number of vertices of $G$. The Merrifield-Simmons index of $G$ is defined as

$$
\sigma=\sigma(G)=\sum_{k \geq 0} n(G, k) .
$$

For detailed information on the mathematical properties of $\sigma$ we refer to [11].

A fundamental problem in chemical graph theory consists in finding the extremal values of a topological index over a significant set of graphs. For instance, for trees with exactly one branching vertex (i.e. starlike trees), the problem was solved for the

Key words and phrases. Merrifield-Simmons index, trees, branching vertices.

2010 Mathematics Subject Classification. Primary: 05C69, 05C35. Secondary: 05 C05.

Received: August 20, 2016.

Accepted: January 18, 2017. 
Wiener index [3], the Hosoya index [4], the Randić index or more generally, for vertexdegree-based topological indices [1]. Moreover, the extremal values of the Hosoya index over trees with exactly 2 branching vertices can be deduced from [6]. See also [5] for the Wiener index.

Let $\Omega(n, i)$ denote the set of all trees with $n$ vertices and $i$ branching vertices. Note that in $\Omega(n, 1)$ (i.e., the set of starlike trees), the star maximizes $\sigma$ [8] and the starlike tree $T_{2,2, n-5}$ (two branches of length 2 and one branch of length $n-5$ ) minimizes $\sigma$ [12]. So it is natural to consider the question: which trees in $\Omega(n, 2)$ minimize and maximize $\sigma$ ? Denoting by $S\left(a_{1}, \ldots, a_{r} ; \mathbf{t} ; b_{1}, \ldots, b_{s}\right)$ the tree with two branching vertices of degrees $r+1, s+1>2$ connected by the path $P_{t}$, and in which the lengths of the pendent paths attached to the two branching vertices are $a_{1}, \ldots, a_{r}$ and $b_{1}, \ldots, b_{s}$ respectively (see Figure 1). We show in Theorems 2.1 and 2.5 that among all trees in $\Omega(n, 2)$, the tree $S(\underbrace{1, \ldots, 1}_{n-4} ; \mathbf{2} ; 1,1)$ maximizes $\sigma$ and the tree $S(n-8,2 ; \mathbf{2} ; 2,2)$ minimizes $\sigma$.

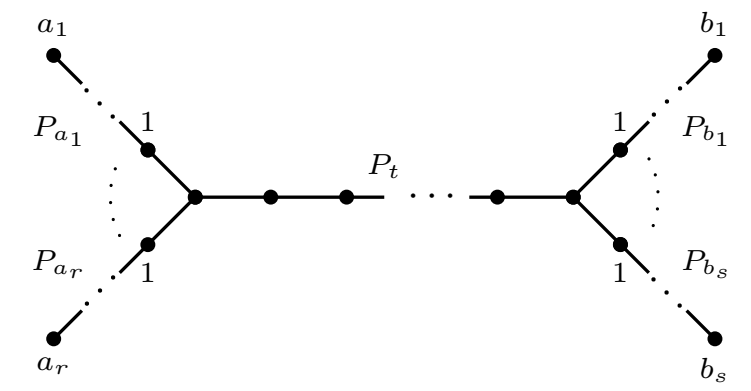

Figure 1 . The tree $S\left(a_{1}, \ldots, a_{r} ; \mathbf{t} ; b_{1}, \ldots, b_{s}\right)$ in $\Omega(n, 2)$.

For each integer $t \geq 2$, we also consider the set $\Omega^{t}(n, 2)$ of all trees in $\Omega(n, 2)$ such that the branching vertices are connected by the path $P_{t}$. We show in Theorems 2.6 and 2.7 that among all trees in $\Omega^{t}(n, 2)$, the tree $S(1,1 ; \mathbf{t} ; \underbrace{1, \ldots, 1}_{n-t-2})$ maximizes $\sigma$ and the tree $S(2,2 ; \mathbf{t} ; 2, n-t-6)$ minimizes $\sigma$.

\section{Extremal Values of the Merrifield-Simmons Index for Trees With Two Branching Vertices}

The following relations for the Merrifield-Simmons index are fundamental and can be found in [7]:

a) if $G_{1}, \ldots, G_{r}$ are the connected components of the graph $G$, then

$$
\sigma(G)=\prod_{i=1}^{r} \sigma\left(G_{i}\right)
$$


b) if $v$ is a vertex of $G$, then

$$
\sigma(G)=\sigma(G-v)+\sigma\left(G-N_{G}[v]\right)
$$

where $N_{G}[v]=\{v\} \cup\{u \in V(G): u v \in E(G)\}$.

Let $G$ and $H$ be two graphs and $u \in V(G), v \in V(H)$. We denote by $G(u, v) H$ the coalescence of $G$ and $H$ at the vertices $u$ and $v$.

Let $P_{n}, S_{n}$ and $T_{n}$ be the path, the star and an arbitrary tree with $n$ vertices respectively and consider arbitrary connected graphs $X$ and $A$ with at least two vertices. If $\{1,2, \ldots, n\}$ are the vertices of $P_{n}, s$ is the central vertex of $S_{n}$ and $t, x$ and $a$ are vertices of $T_{n}, X$ and $A$ respectively, we define the coalescence graphs $X P_{n}=P_{n}(1, x) X, X T_{n}=T_{n}(t, x) X, X S_{n}=S_{n}(s, x) X, X_{n, i}=P_{n}(i, x) X$ and $A X_{n, i}=A(a, n) X_{n, i}$, where the last two graphs are defined for each $i=1, \ldots, n$ (see Figure 2).
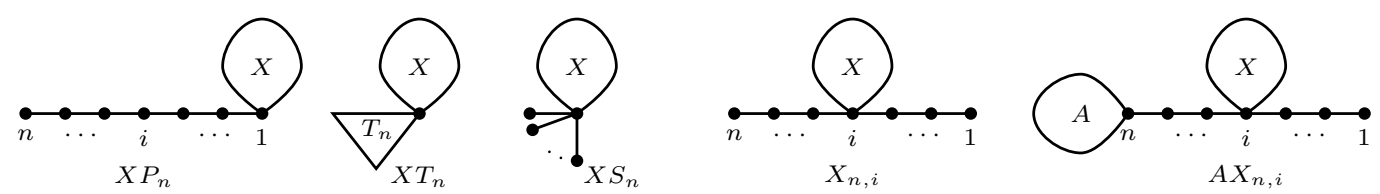

FIGURE 2. Some special graphs

The following results plays a major role in the analysis of treelike graphs and will be used in the sequel.

Lemma 2.1. [11, Theorem 15] Let $X$ be a connected graph, $x \in V(X)$ and $T_{n}$ any tree of order $n$. Then

$$
\sigma\left(X P_{n}\right) \leq \sigma\left(X T_{n}\right) \leq \sigma\left(X S_{n}\right)
$$

Lemma 2.2. [12, Theorem 1] Let $X$ be a connected graph with at least two vertices and $x \in V(X)$ : Let $n=4 m+i$ where $i \in\{1,2,3,4\}$. Then

$$
\begin{aligned}
\sigma\left(X_{n, 2}\right) & >\sigma\left(X_{n, 4}\right)>\cdots>\sigma\left(X_{n, 2 m+2 l}\right) \\
& >\sigma\left(X_{n, 2 m+1}\right)>\cdots>\sigma\left(X_{n, 5}\right)>\sigma\left(X_{n, 3}\right)>\sigma\left(X_{n, 1}\right),
\end{aligned}
$$

where $l=\left\lfloor\frac{i-1}{2}\right\rfloor$.

Our first auxiliary result is of great importance in our work.

Lemma 2.3. Let $A$ and $X$ be a connected graphs with at least two vertices. Then

$$
\sigma\left(A X_{n, i}\right)>\sigma\left(A X_{n, 3}\right)
$$

for all $2 \leq i \leq n-2$ and $i \neq 3$. 
Proof. For $A X_{n, i}=A(a, n) X_{n, i}$ we denote by $x$ the vertex obtained by identifying $a$ and $n$. Then for every $2 \leq i \leq n-2$ we have

$$
\begin{aligned}
\sigma\left(A X_{n, i}\right)-\sigma\left(A X_{n, 3}\right)= & \sigma(A-x)\left[\sigma\left(X_{n-1, i}\right)-\sigma\left(X_{n-1,3}\right)\right] \\
& +\sigma\left(A-N_{A}[x]\right)\left[\sigma\left(X_{n-2, i}\right)-\sigma\left(X_{n-2,3}\right)\right] .
\end{aligned}
$$

The result follows from Lemma 2.2 .

We first consider the problem of finding the tree in $\Omega(n, 2)$ with maximal value of the Merrifield-Simmons index.

Lemma 2.4. Let $t, p, q \geq 2$ be integers such that $p \leq q$. Then

$$
\sigma(S(\underbrace{1, \ldots, 1}_{p} ; \mathbf{t} ; \underbrace{1, \ldots, 1}_{q}))<\sigma(S(\underbrace{1, \ldots, 1}_{p-1} ; \mathbf{t} ; \underbrace{1, \ldots, 1}_{q+1})) .
$$

Proof. Let $U=S(\underbrace{1, \ldots, 1}_{p} ; \mathbf{t} ; \underbrace{1, \ldots, 1}_{q})$ and $V=S(\underbrace{1, \ldots, 1}_{p-1} ; \mathbf{t} ; \underbrace{1, \ldots, 1}_{q+1})$.

If $t=2$, using relations (2.1) and (2.2) we have

$$
\begin{aligned}
& \sigma(U)=\sigma(S(\underbrace{1, \ldots, 1}_{p-1} ; \mathbf{2} ; \underbrace{1, \ldots, 1}_{q}))+2^{p-1} \sigma\left(S_{q+1}\right), \\
& \sigma(V)=\sigma(S(\underbrace{1, \ldots, 1}_{p-1} ; \mathbf{2} ; \underbrace{1, \ldots, 1}_{q}))+2^{q} \sigma\left(S_{p}\right),
\end{aligned}
$$

where as usual $S_{n}$ denotes the star graph of order $n$. Therefore

$\sigma(V)-\sigma(U)=2^{q} \sigma\left(S_{p}\right)-2^{p-1} \sigma\left(S_{q+1}\right)=2^{q}\left(2^{p-1}+1\right)-2^{p-1}\left(2^{q}+1\right)=2^{q}-2^{p-1}>0$.

If $t \geq 3$, using relations (2.1) and (2.2) we obtain

$$
\begin{aligned}
& \sigma(U)=\sigma(S(\underbrace{1, \ldots, 1}_{p-1} ; \mathbf{t} ; \underbrace{1, \ldots, 1}_{q}))+2^{q+p-1} \sigma\left(P_{\mathbf{t}-2}\right)+2^{p-1} \sigma\left(P_{\mathbf{t}-3}\right) ; \\
& \sigma(V)=\sigma(S(\underbrace{1, \ldots, 1}_{p-1} ; \mathbf{t} ; \underbrace{1, \ldots, 1}_{q}))+2^{q+p-1} \sigma\left(P_{\mathbf{t}-2}\right)+2^{q} \sigma\left(P_{\mathbf{t}-3}\right) .
\end{aligned}
$$

Therefore, $\sigma(V)-\sigma(U)=\left(2^{q}-2^{p-1}\right) \sigma\left(P_{\mathbf{t}-3}\right)>0$.

Theorem 2.1. Let $n \geq 7$ and $T=S\left(a_{1}, \ldots, a_{r} ; \mathbf{t} ; b_{1}, \ldots, b_{s}\right) \in \Omega(n, 2)$ where $\mathbf{t} \geq 2$. Then

$$
\sigma(T) \leq \sigma(S(\underbrace{1, \ldots, 1}_{n-4} ; \mathbf{2} ; 1,1))
$$

Proof. By Lemma 2.1 we have that

$$
\sigma(T) \leq \sigma\left(S(a_{1}, \ldots, a_{r} ; \mathbf{2} ; \underbrace{1, \ldots, 1}_{s^{\prime}})\right) \leq \sigma\left(S(\underbrace{1, \ldots, 1}_{r^{\prime}} ; \mathbf{2} ; \underbrace{1, \ldots, 1}_{s^{\prime}})\right),
$$


where $s^{\prime}=t-2+\sum_{j=1}^{s} b_{j} \geq 2$ and $r^{\prime}=\sum_{i=1}^{r} a_{i} \geq 2$. Applying Lemma 2.4 we deduce that

$$
\sigma\left(S(\underbrace{1, \ldots, 1}_{r^{\prime}} ; \mathbf{2} ; \underbrace{1, \ldots, 1}_{s^{\prime}})\right) \leq \sigma(S(\underbrace{1, \ldots, 1}_{n-4} ; \mathbf{2} ; 1,1))
$$

and the result follows.

In what follows we will consider the problem of finding the tree in $\Omega(n, 2)$ with minimal Merrifield-Simmons index.

Let $n>10$ and $T=S\left(a_{1}, \ldots, a_{r} ; \mathbf{t} ; b_{1}, \ldots, b_{s}\right)$ in $\Omega(n, 2)$. By Lemma 2.1

$$
\sigma\left(S\left(a_{1}, \ldots, a_{r} ; \mathbf{t} ; b_{1}, \ldots, b_{s}\right)\right) \geq \sigma\left(S\left(a_{1}, \ldots, a_{r} ; \mathbf{t} ; s^{\prime \prime}, b_{s}\right)\right) \geq \sigma\left(S\left(r^{\prime \prime}, a_{r} ; \mathbf{t} ; s^{\prime \prime}, b_{s}\right)\right),
$$

where $s^{\prime \prime}=\sum_{j=1}^{s-1} b_{j}$ and $r^{\prime \prime}=\sum_{i=1}^{r-1} a_{i}$. Therefore, in order to find the tree with minimal Merrifield-Simmons index for the class $\Omega(n, 2)$, it is enough to find the tree with minimal Merrifield-Simmons index for the subclass of $\Omega(n, 2)$ consisting of all trees of the form $T=S(w, x ; \mathbf{t} ; y, z)$, where $w, x, y, z \geq 1$ are integers.

Next we find the tree with minimal Merrifield-Simmons index over the sets of trees of the form $S(w, x ; \mathbf{t} ; y, z)$ with $\mathbf{t}>2$.

Theorem 2.2. Let $n>10$ and $T=S(w, x ; \mathbf{t} ; y, z)$ where $\mathbf{t}>2$. Then

$$
\sigma(T) \geq \sigma(S(2,2 ; \mathbf{n}-\mathbf{8} ; 2,2)) \text {. }
$$

Proof. Assume first that $w+x \geq 4$. Then by Lemma 2.2 we obtain

$$
\sigma(T) \geq \sigma(S(w+x-2,2 ; \mathbf{t} ; y, z)) .
$$

Moreover $\mathbf{t}>2$ implies that $w+x-2+\mathbf{t}>4$ then we can use Lemma 2.3 to obtain

$$
\sigma(S(w+x-2,2 ; \mathbf{t} ; y, z)) \geq \sigma(S(2,2 ; \mathbf{t}+\mathbf{w}+\mathbf{x}-\mathbf{4} ; y, z)) .
$$

Now if $y+z \geq 4$, then a similar argument ends the proof (see Figure 3 ). Otherwise $y+z \leq 3$ which implies that $S(2,2 ; \mathbf{t}+\mathbf{w}+\mathbf{x}-\mathbf{4} ; y, z)$ is the tree $S(2,2 ; \mathbf{n}-\mathbf{7} ; 1,2)$ or the tree $S(2,2 ; \mathbf{n}-\mathbf{6} ; 1,1)$. Since $n>10$, we have that $n-6>n-7>3$ and in both cases the result follows using Lemma 2.3.

The only case left to consider is when $w+x \leq 3$ and $y+z \leq 3$, but in this situation we note that necessarily $\mathbf{t}>4$ and the result follows using Lemma 2.3.

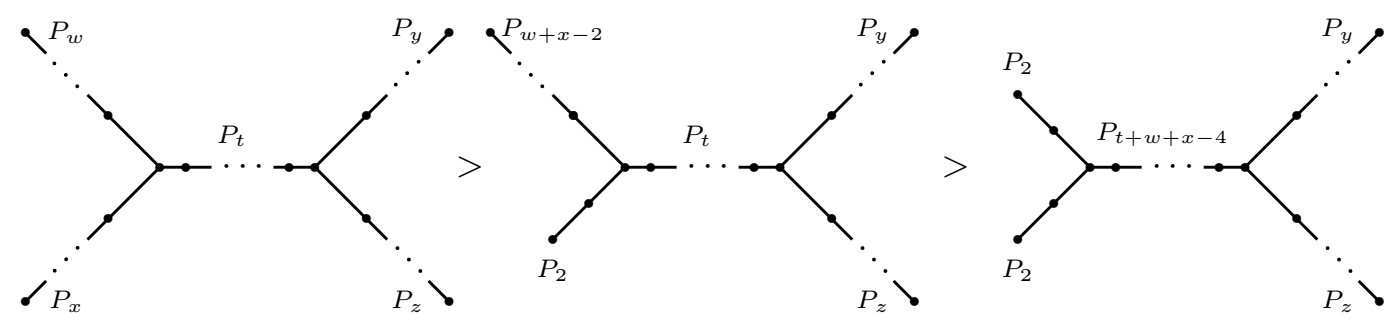

FiguRE 3. Graphs in the proof of Theorem 2.2 
Lemma 2.5. Let $\mathbf{t}, w \geq 2$ be integers. Then

$$
\sigma(S(w, 2 ; \mathbf{t} ; 2,2))<\sigma(S(w+1,2 ; \mathbf{t} ; 1,2)) .
$$

Proof. Let $A=S(w, 2 ; \mathbf{t} ; 2,2)$ and $B=S(w+1,2 ; \mathbf{t} ; 1,2)$. Using relations (2.1), (2.2) and Lemma 2.1 we have

$$
\begin{aligned}
\sigma(A) & \left.=\sigma(S(w, 2 ; \mathbf{t} ; 1,2))+\sigma\left(T_{w, 2, \mathbf{t}+\mathbf{1}}\right)\right), \\
\sigma(B) & =\sigma(S(w, 2 ; \mathbf{t} ; 1,2))+\sigma(S(w-1,2 ; \mathbf{t} ; 1,2)) \\
& \left.\geq \sigma(S(w, 2 ; \mathbf{t} ; 1,2))+\sigma\left(T_{w+\mathbf{t + 1 , 2 , 1}}\right)\right),
\end{aligned}
$$

where $T_{a, b, c}$ is a starlike tree with branches of length $a, b$ and $c$ respectively and $a+b+c+1=n$. Hence

$$
\left.\left.\sigma(B)-\sigma(A) \geq \sigma\left(T_{w+\mathbf{t}+\mathbf{1}, \mathbf{2}, \mathbf{1}}\right)\right)-\sigma\left(T_{w, 2, \mathbf{t}+\mathbf{1}}\right)\right)>0
$$

by Lemma 2.2 .

Lemma 2.6. Let $\mathbf{t}, w, y$ be integers such that $\mathbf{t} \geq 2$ and $w \geq y \geq 2$. If $y$ is odd then

$$
\sigma(S(w, 2 ; \mathbf{t} ; y, 2))>\sigma(S(w+1,2 ; \mathbf{t} ; y-1,2)) .
$$

If $y$ is even then

$$
\sigma(S(w, 2 ; \mathbf{t} ; y, 2))<\sigma(S(w+1,2 ; \mathbf{t} ; y-1,2)) .
$$

Proof. Let $A=S(w, 2 ; \mathbf{t} ; y, 2)$ and $B=S(w+1,2 ; \mathbf{t} ; y-1,2)$. Using relations (2.1) and (2.2) we have

$$
\sigma(A)=\sigma(S(w, 2 ; \mathbf{t} ; y-1,2))+\sigma(S(w, 2 ; \mathbf{t} ; y-2,2))
$$

and

$$
\sigma(B)=\sigma(S(w, 2 ; \mathbf{t} ; y-1,2))+\sigma(S(w-1,2 ; \mathbf{t} ; y-1,2)) .
$$

Hence

$$
\sigma(B)-\sigma(A)=(-1)[\sigma(S(w, 2 ; \mathbf{t} ; y-2,2))-\sigma(S(w-1,2 ; \mathbf{t} ; y-1,2))] .
$$

Repeating this argument $y-2$ times we deduce

$$
\sigma(B)-\sigma(A)=(-1)^{y-2}[\sigma(S(w-y+3,2 ; \mathbf{t} ; 1,2))-\sigma(S(w-y+2,2 ; \mathbf{t} ; 2,2))] .
$$

By Lemma 2.5 we know that $\sigma(S(w-y+3,2 ; \mathbf{t} ; 1,2))>\sigma(S(w-y+2,2 ; \mathbf{t} ; 2,2))$ and the result follows.

Theorem 2.3. Let $M=4 k+i$, where $i \in\{0,1,2,3\}$. Then

$$
\begin{aligned}
\sigma\left(G\left(P_{M-2}, P_{2}\right)\right) & <\cdots<\sigma\left(G\left(P_{M-2 k}, P_{2 k}\right)\right) \leq \sigma\left(G\left(P_{M-(2 k+1)}, P_{2 k+1}\right)\right) \\
& <\sigma\left(G\left(P_{M-(2 k-1)}, P_{2 k-1}\right)\right)<\cdots<\sigma\left(G\left(P_{M-1}, P_{1}\right)\right),
\end{aligned}
$$

where $G\left(P_{a}, P_{b}\right)=S(a, 2 ; \mathbf{t} ; b, 2)$, that is $G\left(P_{a}, P_{b}\right)$ is the tree obtained from the path $P_{\mathbf{t}+4}=v_{1} v_{2} \cdots v_{\mathbf{t}+4}$ by joining the path $P_{a}$ to the vertex $v_{3}$ and joining the path $P_{b}$ to the vertex $v_{\mathbf{t}+2}$ (see Figure 4 ). 


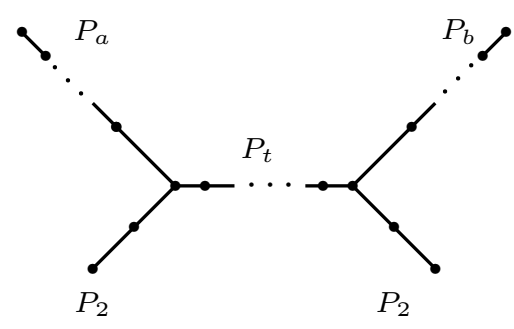

Figure 4. Trees $G(a, b)$.

Proof. Let $A=G\left(P_{a}, P_{b}\right)$ and $B=G\left(P_{a-2}, P_{b+2}\right)$, where $2 \leq b \leq a-4$. Using relations (2.1) and (2.2) we have

$$
\sigma(A)=\sigma\left(G\left(P_{a-1}, P_{b}\right)\right)+\sigma\left(G\left(P_{a-2}, P_{b}\right)\right)
$$

and

$$
\sigma(B)=\sigma\left(G\left(P_{a-2}, P_{b+1}\right)\right)+\sigma\left(G\left(P_{a-2}, P_{b}\right)\right)
$$

Consequently

$$
\sigma(A)-\sigma(B)=(-1)\left[\sigma\left(G\left(P_{a-2}, P_{b+1}\right)\right)-\sigma\left(G\left(P_{a-1}, P_{b}\right)\right)\right]
$$

and so

$$
\sigma(A)-\sigma(B)=(-1)^{b}\left[\sigma\left(G\left(P_{a-b-1}, P_{2}\right)\right)-\sigma\left(G\left(P_{a-b}, P_{1}\right)\right)\right] .
$$

By Lemma 2.5, if $b$ is even then $\sigma(A)<\sigma(B)$ and if $b$ is odd then $\sigma(A)>\sigma(B)$. Only remains to prove that $\sigma\left(G\left(P_{M-2 k}, P_{2 k}\right)\right) \leq \sigma\left(G\left(P_{M-(2 k+1)}, P_{2 k+1}\right)\right)$, but this is a direct consequence of Lemma 2.6.

Lemma 2.7. Let $n>10$ and let $w, x$ be positive integers. Then

$$
\sigma(S(w, x ; \mathbf{2} ; 1,1))>\sigma(S(n-8,2 ; \mathbf{2} ; 2,2)) .
$$

Proof. Let $A=S(w, x ; \mathbf{2} ; 1,1)$ and $B=S(n-8,2 ; \mathbf{2} ; 2,2)$. Since $n>10$ we have that $w+x>6$ and by Lemma 2.2 we can construct a tree $A_{1}=S(n-6,2 ; \mathbf{2} ; 1,1) \in \Omega(n, 2)$ such that $\sigma(A)>\sigma\left(A_{1}\right)$. By a direct computation using relations (2.1) and (2.2) we obtain

$$
\sigma\left(A_{1}\right)=8 \sigma\left(P_{n-7}\right)+15 \sigma\left(P_{n-6}\right)
$$

and

$$
\sigma(B)=18 \sigma\left(P_{n-9}\right)+39 \sigma\left(P_{n-8}\right)
$$

Therefore

$$
\sigma\left(A_{1}\right)-\sigma(B)=4 \sigma\left(P_{n-9}\right)+\sigma\left(P_{n-10}\right)>0
$$

and the result follows. 
Next we find the tree with minimal Merrifield-Simmons index over the sets of trees of the form $S(w, x ; \mathbf{2} ; y, z)$.

Theorem 2.4. Let $n>10$ and $T=S(w, x ; \mathbf{2} ; y, z)$. Then

$$
\sigma(T) \leq \sigma(S(n-8,2 ; \mathbf{2} ; 2,2)) .
$$

Proof. Note that $w+x+y+z>8$. Therefore we may assume without loosing generality that $w+x \geq 4$. Then by Lemma 2.2 there exists a tree $\left.T_{1}=S(w+x-2,2 ; \mathbf{2} ; y, z)\right)$ such that $\sigma(T) \leq \sigma\left(T_{1}\right)$.

If $y+z \geq 4$, by Lemma 2.2 we construct a tree $T_{2}=S(w+x-2,2 ; \mathbf{2} ; y+z-2,2)$ such that $\sigma\left(T_{1}\right)>\sigma\left(T_{2}\right)$ and the result follows from Theorem 2.3.

If $y+z \leq 3$ then $T_{1}=S(w+x-2,2 ; \mathbf{2} ; 1,2)$ or $T_{1}=S(w+x-2,2 ; \mathbf{2} ; 1,1)$. If $T_{1}=S(w+x-2,2 ; \mathbf{2} ; 1,2)$ the result follows from Theorem 2.3. On the other hand, if $T_{1}=S(w+x-2,2 ; \mathbf{2} ; 1,1)$ the result follows from Lemma 2.7 .

In our next result we find the minimal tree with respect to Merrifield-Simmons index over $\Omega(n, 2)$.

Theorem 2.5. For every $n \geq 11, S(n-8,2 ; \mathbf{2} ; 2,2)$ is the tree with minimal MerrifieldSimmons index in $\Omega(n, 2)$.

Proof. Bearing in mind Theorems 2.2 and 2.4 to obtain the result it is enought to compare the Merrifiel-Simmons index for the trees $S(2,2 ; \mathbf{n}-\mathbf{8} ; 2,2)$ and $S(n-$ $8,2 ; \mathbf{2} ; 2,2)$. Indeed, let $A=S(2,2 ; \mathbf{n}-\mathbf{8} ; 2,2)$ and let $B=S(n-8,2 ; \mathbf{2} ; 2,2)$. By a direct computation, using relations (2.1) and (2.2), we obtain

$$
\begin{aligned}
\sigma(A) & =81 \sigma\left(P_{n-10}\right)+72 \sigma\left(P_{n-11}\right)+16 \sigma\left(P_{n-12}\right) \\
& =41 \sigma\left(P_{n-8}\right)+15 \sigma\left(P_{n-9}\right),
\end{aligned}
$$

and

$$
\sigma(B)=39 \sigma\left(P_{n-8}\right)+18 \sigma\left(P_{n-9}\right) .
$$

Hence

$$
\sigma(A)-\sigma(B)=2 \sigma\left(P_{n-10}\right)-\sigma\left(P_{n-9}\right)>0 ;
$$

and the result follows.

To end this section we consider the problem of finding extremal values of the Merrifield-Simmons index for trees with two branching vertices at a fixed distance. Consider the set $\Omega^{\mathrm{t}}(n, 2)$ of all trees in $\Omega(n, 2)$ such that the two branching vertices are connected by the path $P_{t}$; that is, the distance between the two branching vertices is $t-1$. We next find the extremal trees in $\Omega^{\mathbf{t}}(n, 2)$ with respect to the MerrifieldSimmons index.

Theorem 2.6. Let $n \geq \mathbf{t}+4$ and $T \in \Omega^{\mathbf{t}}(n, 2), T \neq S(1,1 ; \mathbf{t} ; \underbrace{1, \ldots, 1}_{n-\mathbf{t}-2})$. Then

$$
\sigma(T)<\sigma(S(1,1 ; \mathbf{t} ; \underbrace{1, \ldots, 1}_{n-\mathbf{t}-2})) .
$$


Proof. By Lemma 2.1 it is sufficient to consider trees in $\Omega^{\mathbf{t}}(n, 2)$ of the form $T=$ $S(\underbrace{1, \ldots, 1}_{p} ; \mathbf{t} ; \underbrace{1, \ldots, 1}_{q})$. We may assume that $p \leq q$. Now, a repeated application of Lemma 2.4 gives that $\sigma(T)<\sigma(S(1,1 ; \mathbf{t} ; \underbrace{1, \ldots, 1}_{n-\mathbf{t}-2}))$.

Theorem 2.7. Let $n \geq \mathbf{t}+7$ and $T \in \Omega^{\mathbf{t}}(n, 2), T \neq S(2,2 ; \mathbf{t} ; 2, n-\mathbf{t}-6)$. Then

$$
\sigma(T)>\sigma(S(2,2 ; \mathbf{t} ; 2, n-\mathbf{t}-6)) .
$$

Proof. Bearing in mind Theorem 2.4 and Lemma 2.1, it is clear that in order to obtain the result it is enough to consider the case $\mathbf{t} \geq 3$ and trees in $\Omega^{\mathbf{t}}(n, 2)$ of the form $T=S(w, x ; \mathbf{t} ; y, z)$.

Note that $w+x+y+z \geq 7$. Therefore as in the proof of Theorem 2.2, there exists a tree $T_{1} \in \Omega^{\mathbf{t}}(n, 2)$ of the form $T_{1}=S(r, 2, ; \mathbf{t} ; s, 2)$ such that $\sigma(T)>\sigma\left(T_{1}\right)$, where $r+s=n-\mathbf{t}-4$. Note that $T_{1}=G\left(P_{r}, P_{s}\right)$, therefore the result follows from Theorem 2.3 .

\section{REFERENCES}

[1] C. Betancur, R. Cruz and J. Rada, Vertex-degree-based topological indices over starlike trees, Discrete Appl. Math. 185 (2015), 18-25.

[2] J. Devillers and A. T. Balaban (Eds.), Topological Indices and Related Descriptors in: QSAR and QSPR, Gordon \& Breach, Amsterdam, 1999.

[3] I. Gutman, J. Rada and O. Araujo, The Wiener index of starlike trees and a related partial order, MATCH Commun. Math. Comput. Chem. 42 (2000), 145-154.

[4] I. Gutman, O. Araujo and J. Rada, Matchings in starlike trees, Appl. Math. Lett. 14 (2001), 843-848.

[5] H. Lin, On the Wiener index of trees with given number of branching vertices, MATCH Commun. Math. Comput. Chem 72 (2014), 301-310.

[6] C. Marín, J. Monsalve and J. Rada, Maximum and minimum energy trees with two and three branched vertices, MATCH Commun. Math. Comput. Chem. 74(2) (2015), 285-306.

[7] R. E. Merrifield and H. E. Simmons. Topological Methods in Chemistry, Wiley, New York, 1989.

[8] H. Prodinger and R. F. Tichy, Fibonacci numbers of graphs, Fibonacci Quart. 20(1) (1982), 16-21.

[9] R. Todeschini and V. Consonni, Handbook of Molecular Descriptors, Wiley-VCH, Weinheim, 2000 .

[10] R. Todeschini and V. Consonni, Molecular Descriptors for Chemoinformatics, Vols. 1 \& 2, Wiley-VCH, Weinheim, 2009.

[11] S. Wagner and I. Gutman, Maxima and minima of the Hosoya index and the Merrifield-Simmons index: a survey of results and techniques, Acta Appl. Math. 112 (2010), 323-346.

[12] H. Zhao and X. Li, On the Fibonacci numbers of trees, Fibonacci Quart. 44(1) (2006), 32-38. 
${ }^{1}$ Instituto de Matemáticas, UniversidAd DE ANTIOQUiA

Medellín, Colombia.

E-mail address: roberto.cruz@udea.edu.co

${ }^{2}$ Instituto de Matemáticas

UNIVERSIDAD DE ANTIOQUiA

Medellín, Colombia.

E-mail address: calberto.marin@udea.edu.co

${ }^{3}$ Instituto de Matemáticas

Universidad DE Antioquia

Medellín, Colombia.

E-mail address: pablo.rada@udea.edu.co 